\title{
Springer
}

Available online at https://link.springer.com/journal/42241

http://www.jhydrodynamics.com

Journal of Hydrodynamics, 2019, 31(1): 774-781

https://doi.org/10.1007/s44241-000-0000-0

\section{Development of a fully coupled aero-hydro-mooring-elastic tool for floating offshore wind turbines"}

\author{
Yuanchuan Liu, Qing Xiao \\ Department of Naval Architecture, Ocean and Marine Engineering, University of Strathclyde, Glasgow, UK
}

(Received November 1, 2018, Revised November 28, 2018, Accepted December 2, 2018, Published online)

(C) China Ship Scientific Research Center 2019

\begin{abstract}
A floating offshore wind turbine (FOWT) is a coupled system where a wind turbine with flexible blades interacts with a moored platform in wind and waves. This paper presents a high-fidelity aero-hydro-mooring-elastic analysis tool developed for FOWT applications. A fully coupled analysis is carried out for an OC4 semi-submersible FOWT under a combined wind/wave condition. Responses of the FOWT are investigated in terms of platform hydrodynamics, mooring dynamics, wind turbine aerodynamics and blade structural dynamics. Interactions between the FOWT and fluid flow are also analysed by visualising results obtained via the CFD approach. Through this work, the capabilities of the tool developed are demonstrated and impacts of different parts of the system on each other are investigated.
\end{abstract}

Key words: Floating offshore wind turbine, fluid-structure interaction, blade elasticity, mooring dynamics, OpenFOAM

\section{Introduction}

As one of the fastest growing renewable energy sources, wind energy is playing an increasingly important role in addressing the issues of climate change and energy crisis the world is currently facing. To extract energy from wind, wind turbines are traditionally installed onshore in rural open fields. Over the past several decades, an increasing number of wind turbines are installed in offshore areas where wind resource is more abundant compared with onshore sites. It is predicted that by 2020 the cumulative installed capacity of offshore wind in the EU will significantly increase to $24.6 \mathrm{GW}$ from $12.6 \mathrm{GW}$ in $2016^{[1]}$. In the last few years, several floating wind projects ${ }^{[2-4]}$ have also emerged by installing wind turbines far offshore in deep-water sites on moored platforms.

The abundance of wind resource far offshore and the vast deep-water sites suitable for installation make floating wind turbines very promising. However, compared to their land-based or offshore fixed-bottom counterparts, challenges are present for floating offshore wind turbines (FOWT) in terms of engineering design and analysis. An FOWT is a fully coupled system operating under complex environmental conditions, such as wind and waves, where a wind turbine with flexible blades and a floating platform with its mooring system supporting the turbine interact with each other. On one hand, apart from the dynamic loadings from waves and the mooring system directly applied to the platform, the wind loading exerted upon the turbine also contributes to the overall system loading, and thus influences the dynamic six degreesof-freedom (6DOF) motion responses of the floating system. On the other hand, the $6 \mathrm{DOF}$ motions of the system change the position and orientation of the wind turbine, which in turn exert impacts on its aerodynamic performance and subsequently the dynamic structural responses of its aeroelastic blades, leading to severe structural problems such as fatigue. As traditional design tools targeting fixed-bottom wind turbines are unable to take into consideration the complex interactions within an FOWT during the design process, it is thus essential to develop fully coupled aero-hydromooring-elastic tools, which are capable of performing fluid-structure interaction (FSI) analysis for FOWTs under various operating conditions ${ }^{[5]}$.

In recent years, a number of tools with the capability of carrying out fully coupled analysis for FOWTs have shown up. Examples are the well-known fatigue, aerodynamics, structures, and turbulence

\footnotetext{
* Biography: Yuanchuan Liu (1990-), Male, Ph. D., E-mail: liuyuanchuan456@163.com Corresponding author: Qing Xiao,
} 
(FAST) code from National Renewable Energy Laboratory (NREL) of the USA, HAWC2 developed at Technical University of Denmark (DTU) and the commercial software package GH-Bladed developed by Garrad Hassan (GH) Ltd. These tools are highly efficient and are suitable for the initial design stage when a large number of cases need to be simulated. A large number of fully coupled FOWT studies have been performed using these tools ${ }^{[6-9]}$. Although they are computationally efficient, the engineering models adopted by the tools have some limitations, particularly on aerodynamics and hydrodynamics. For example, the blade element momentum (BEM) theory they utilise to deal with wind turbine aerodynamics requires a series of empirical correction models, which were originally developed for fixed-bottom wind turbine applications and might be unable to consider the dynamic interactions between the turbine and its wake due to platform motions in a floating scenario ${ }^{[10]}$. In terms of platform hydrodynamics, almost all these tools employ the linear potential flow theory ${ }^{[1]}$ and/or Morison's equation. The linear assumption of the potential flow theory makes it inadequate for highly nonlinear problems, such as the cases with large structure motion responses ${ }^{[12]}$. Meanwhile, the effects of fluid viscosity cannot be taken into account in potential-based methods and the approximated viscous drag in Morison's formulation requires an additional calibrated quadratic damping coefficient, which relies on experimental test ${ }^{[3,13]}$.

Meanwhile, computational fluid dynamics (CFD) has recently been applied to FOWT studies by researchers in several different ways due to many of its advantages. Compared with those engineering tools abovementioned, CFD-based approaches can inherently consider viscous and nonlinear effects without empirical corrections and additional input from experiment, and thus are able to provide more accurate results. Besides, CFD methods can be used to visualise and examine detailed flow field at any position to gain insight into sophisticated physical phenomena, such as the interactions between a moving wind turbine and its dynamic wake. Due to the complexity of FOWT analysis, most existing investigations using CFD tools simplified the problem either by focusing on the hydrodynamic responses of a floater without considering loadings from its suppor- ted turbine ${ }^{[13-15]}$ or by studying the aerodynamic performance of a wind turbine while imposing prescri- bed platform motion responses to the turbine ${ }^{[16-18]}$. Although there have been several recent applications of CFD methods to coupled FOWT simulations, these studies mainly focused on the modelling of aerody- namics and hydrodynamics while simplifications were usually made regarding structural flexibility and mooring system analysis ${ }^{[19-21]}$. In their models, turbine blades were assumed to be rigid and structural flexibility were simply neglected.
Furthermore, quasi- static methods were employed for mooring analysis without considering dynamic effects. As a result, further work is still required to develop a fully coupled high-fidelity analysis tool for FOWT applications, including flexible turbine blade modelling and mooring system dynamic analysis.

This paper presents a fully coupled CFD-based aero-hydro-mooring-elastic analysis tool developed for FOWTs under combined wind/wave conditions. This high-fidelity tool can be utilised to help gain a better understanding of the underlying physics and sophisticated interactions between wind/wave and an FOWT as well as the influence of different components on each other within the system. Meanwhile, as a supplementary means to expensive experimental tests, it can also help validate and calibrate existing engineering tools.

\section{Numerical methods}

\subsection{Overview}

The present numerical tool developed for fully coupled FSI analysis of FOWTs is a continuation and an integrated outcome of two previously established codes, i.e., an aero-hydrodynamic analysis code for FOWTs without considering blade elasticity and mooring system dynamics ${ }^{[22]}$, and an aero-elastic code for wind turbines with flexible blades and imposed platform motions ${ }^{[23-24]}$. In this tool, wind turbine aerodynamics and floating platform hydrodynamics are studied using a CFD approach based on an open source CFD toolbox OpenFOAM, which is coupled with an open source MultiBody Dynamics (MBD) code MBDyn utilised to solve the structural dynamics of an FOWT with flexible turbine blades. The coupling between the two codes is achieved by establishing an interface library to exchange force and motion data between these two codes. Additionally, a mesh motion solver is developed in OpenFOAM to tackle complex mesh movement in FOWT simulations. A mooring system analysis module with both quasi- static and dynamic modelling features is also implemented to simulate mooring lines in an FOWT. Figure 1 depicts the structure of the present tool, where built-in features in OpenFOAM and MBDyn are indicated in black; the wave modelling module marked in red is incorporated from a previously developed solver ${ }^{[25-26]}$; new functionalities implemented in this work are highlighted in blue. In the following sections, these numerical techniques are briefly described, and the overall coupling procedure of the tool is also presented.

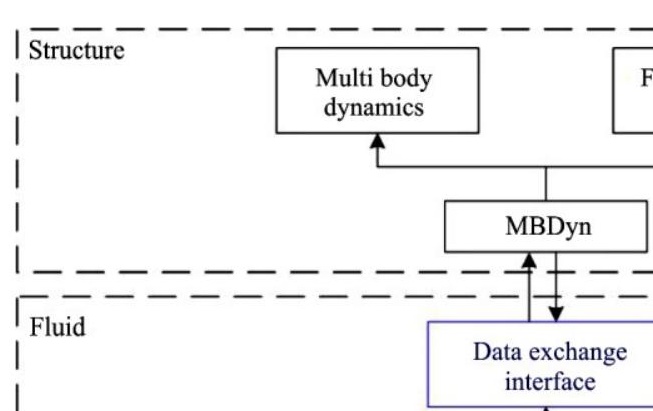




\subsection{Fluid flow modelling}

The fluid flow around an FOWT is assumed to be transient, incompressible and viscous. In this tool, the flow field is obtained in OpenFOAM by solving the following continuity and Reynolds-averaged NavierStokes (RANS) equations in an Arbitrary LagrangianEulerian (ALE) form:

$$
\nabla \cdot \boldsymbol{U}=0
$$

$$
\begin{aligned}
& \frac{\partial \rho \boldsymbol{U}}{\partial t}+\nabla \cdot\left[\rho\left(\boldsymbol{U}-\boldsymbol{U}_{g}\right) \boldsymbol{U}\right]=-\nabla p_{d}-\boldsymbol{g} \cdot \boldsymbol{x} \nabla \rho+ \\
& \nabla \cdot\left(\mu_{\mathrm{ef}} \nabla \boldsymbol{U}\right)+\nabla \boldsymbol{U} \cdot \nabla \mu_{\mathrm{e}}
\end{aligned}
$$

where $\boldsymbol{U}, \boldsymbol{U}_{g}$ represent the velocity of flow field and grid nodes, respectively; $p_{d}=p-\rho \boldsymbol{g} \cdot \boldsymbol{x}$ is the pressure of flow field obtained by subtracting the hydrostatic part $\rho \boldsymbol{g} \cdot \boldsymbol{x}$ from total pressure $p ; \boldsymbol{g}$ is the gravitational acceleration vector; $\rho$ is fluid density, $\mu_{\text {eff }}=\rho\left(v+v_{t}\right)$ denotes the effective dynamic viscosity of fluid, in which $v, v_{t}$ are kinematic and eddy viscosity, separately.

In typical FOWT simulations, Reynolds number can be as high as $10^{7}$ and fluid flow is fully turbulent. A two-equation $k-\omega$ shear stress transport (SST) turbulence model ${ }^{[27]}$ is thus adopted as the closure for the RANS equations. In order to capture the interface or free surface between two fluids, such as air and water in the case of FOWTs, a volume of fluid (VOF) method $^{[28]}$ is employed. A wave generation module previously developed by Professor Decheng Wan's research group in Shanghai Jiao Tong University is incorporated in the present code for wave modelling, which is able to simulate various types of waves including linear wave, Stokes 2nd order wave, freak wave, solitary wave, irregular wave, etc. ${ }^{[25-26]}$. Details about these models can be found in a previous work ${ }^{[22]}$.

\subsection{Structural responses calculation}

The dynamic structural responses of an FOWT are solved with the help of the MBD-based code MBDyn, which adopts a Lagrange multiplier formu- lation for a multibody system consisting of rigid and flexible bodies connected by kinematic constraints ${ }^{[29]}$. For each body of the system, Newton-Euler equations of motion are established while connections between adjacent parts are represented as constraint equations.

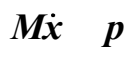

$\dot{\boldsymbol{p}}{ }_{x} \lambda \boldsymbol{f} \boldsymbol{x} \dot{\boldsymbol{x}} t$

$\phi(x, t)=0$ 
where $\boldsymbol{M}$ is an inertia matrix; $\boldsymbol{x}$ represents the generalised coordinates including both translational and rotational parameters in the global reference frame; the dot operator above a variable denotes its derivative to time $t ; \boldsymbol{p}$ is a momentum vector; $\phi$ is a set of kinematic constraints applied to the body and $\phi_{x}^{\mathrm{T}}$ is the Jacobian of $\phi$ with respect to $\boldsymbol{x} ; \boldsymbol{f}$ is an external force vector.

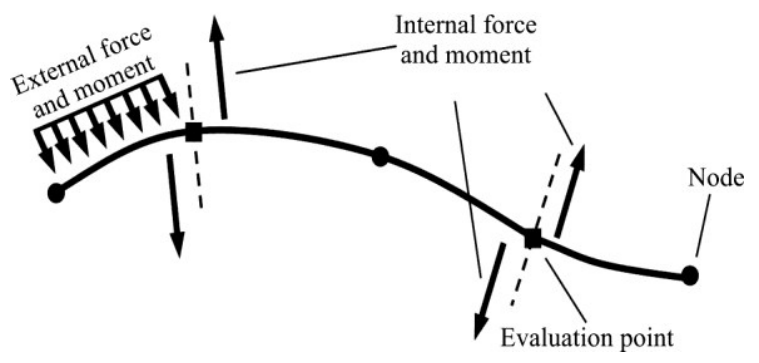

Fig. 2 Illustration of a three-node beam element in MBDyn

An important feature of MBDyn is its capability of modelling flexible bodies, which are discretised as a series of three-node beam elements based on a nonlinear beam theory formulated within a multibody framework ${ }^{[29]}$. As illustrated in Fig. 2, a three-node beam element is divided into three segments by two evaluation points (squares), where internal forces and moments are evaluated via constitutive laws. Each segment is then associated with a node (circles) with lumped mass, for which equilibrium equations are established in a similar way to a normal node representing a rigid body.

\subsection{CFD mesh motion handling}

One of the challenges for simulating an FOWT with flexible blades using a CFD approach is how to handle the motion of the CFD mesh to represent the complex structural responses of the system, which can be categorised into two groups: (1) global rigid body motions, i.e., platform $6 \mathrm{DoF}$ motions and turbine rotation, (2) local flexible body deformation, such as deflections of an elastic turbine blade. The current mesh motion libraries in OpenFOAM are unable to cope with global and local structural responses at the same time. In the present tool, a customised mesh motion library is developed by incorporating features of the built-in solid body motion library into a dynamic mesh motion solver displacementLaplacianFvMotionSolver.

The implemented mesh motion library deals with global rigid body motion responses in a solid body motion manner. The overall computational domain is split into three separate cell zones by two pairs of arbitrary mesh interface (AMI) or sliding mesh surfaces as sketched in Fig. 3. Different rigid body motions of the floating system are then applied to these cell zones as discussed previously ${ }^{[22]}$. When an FOWT is in motion, the outer zone only translates in surge, sway and heave directions. The middle zone experiences three rotational motion responses, i.e., roll, pitch and yaw, as well as the three translational components, while the inner zone undergoes all 6DOF platform motion responses together with a prescribed turbine rotation.

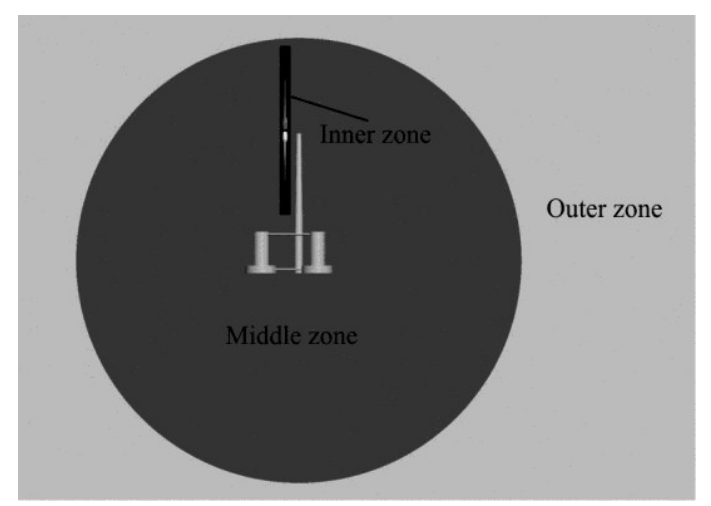

Fig. 3 Cell zone decomposition of computational domain for an FOWT

Meanwhile, mesh motion due to local blade deformation is handled by solving a displacementbased Laplace equation ${ }^{[24]}$ for cell centres inside the inner zone shown in Fig. 3. In order to maintain grid quality, global rigid body motions are firstly subtracted from the displacement of the whole turbine surface computed by MBDyn to obtain a temporary value, which is then used as the boundary condition of the Laplace equation. Once the displacement of cell centres is obtained, interpolation is performed to calculate the displacement of internal grid points, which is then added to the initial position of all points to determine their updated position resulting from blade deformation. Lastly, points in the inner zone are translated and rotated collectively to take into account global rigid body motions.

\subsection{Mooring system analysis}

Mooring systems are essential in station-keeping for floating structures. In this tool, a mooring system analysis module is developed within the OpenFOAM framework to calculate the mooring restoring force provided for an FOWT. A quasi-static method was introduced previously ${ }^{[22]}$ by firstly dividing a mooring line into a number of segments and then establishing equations of static equilibrium for each segment at every time step. The discretised approach utilised by this method enables it to model mooring lines made of multiple components with different structural properties.

In order to take into consideration the dynamic 
effects due to mooring line motions, which are neglected in quasi-static methods, the present mooring system analysis module is further extended by implementing a dynamic method based on a 3-D lumped mass model, in which a mooring line is discretised into $n+1$ concentrated masses (nodes) connected by $n$ massless springs (segments), as illustrated in Fig. 4. Unlike the quasi-static method, dynamic equations of motion are applied to every node in the dynamic approach so that the inertial force associated with mooring line motion is considered and tension force can be accurately predicted.

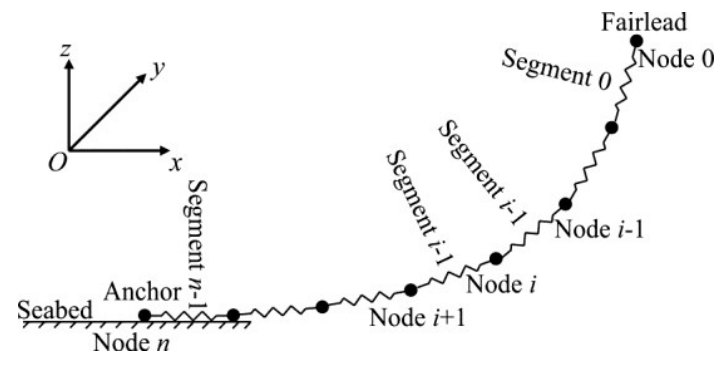

Fig. 4 Sketch of a 3-D lumped mass model
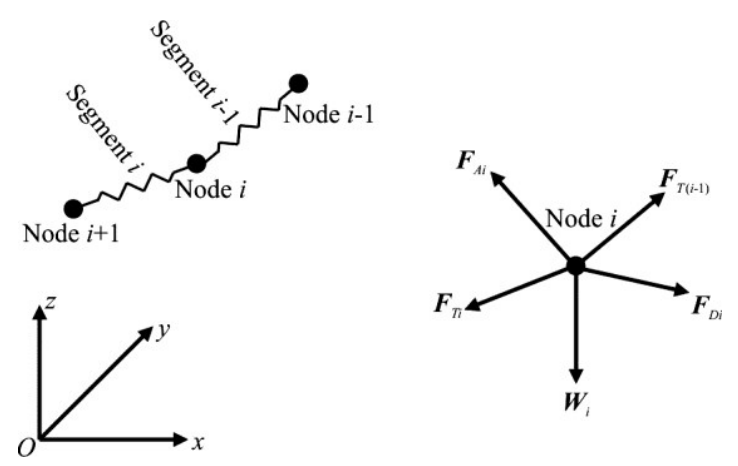

Fig. 5 Sketch of forces exerted on node $i$ in lumped mass method

For node $i$, the various forces applied to it are illustrated in Fig. 5 and its motion is governed by the following equation

$$
m_{i} \ddot{\boldsymbol{X}}_{i} \quad \boldsymbol{F}_{T(i-1)}-\boldsymbol{F}_{T i}+\boldsymbol{F}_{D i}+\boldsymbol{F}_{A i}-\boldsymbol{W}_{i}
$$

where $m_{i}$ denotes mass of node $i ; \ddot{\boldsymbol{X}}_{i}$ represents acceleration vector of node $i ; \boldsymbol{F}_{T(i-1)}$ and $\boldsymbol{F}_{T i}$ are tension force vectors of segments $i-1$ and $i$, respectively; $\boldsymbol{F}_{D i}, \boldsymbol{F}_{A i}$ are hydrodynamic drag and inertia force vectors applied to node $i$, which are approximated via Morison's equation; $\boldsymbol{W}_{i}$ is weight of node $i$ in water.

The Newmark-beta method $(\gamma=0.5, \beta=0.25)$ is employed to solve the differential equations for the motion data of each node. Subsequently, the tension force magnitude $T_{i}$ for segment $i$ shown in Fig. 5 is calculated as

$T_{i}=E_{i} A_{i}\left(\frac{\left|\boldsymbol{X}_{i+1}-\boldsymbol{X}_{i}\right|}{l_{i}}-1\right)$

where $E_{i}, A_{i}$ denote the Young's modulus and cross-sectional area for the segment, separately; $\boldsymbol{X}_{i}$ represents the coordinates of node $i$ in the global reference frame; $l_{i}$ stands for the unstretched or original length of the segment.

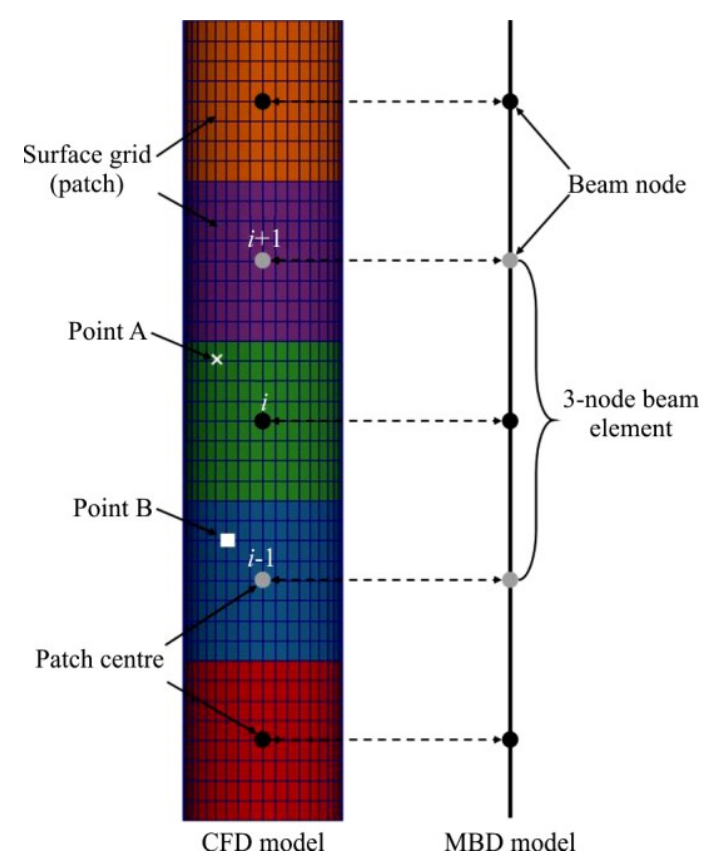

Fig. 6 (Color online) Diagram for mapping between CFD and MBD models ${ }^{[24]}$

\subsection{Coupling procedure}

As mentioned earlier in Section 1.3, a flexible wind turbine blade is simplified as a beam-like structure in MBDyn and modelled as a series of threenode beam elements consisting of geometrical nodes. On the other hand, the same geometry is discretised into a complex surface grid comprising a large number of points in OpenFOAM. These two different representations of the blade lead to a pair of unmatched interfaces. A mapping scheme is therefore

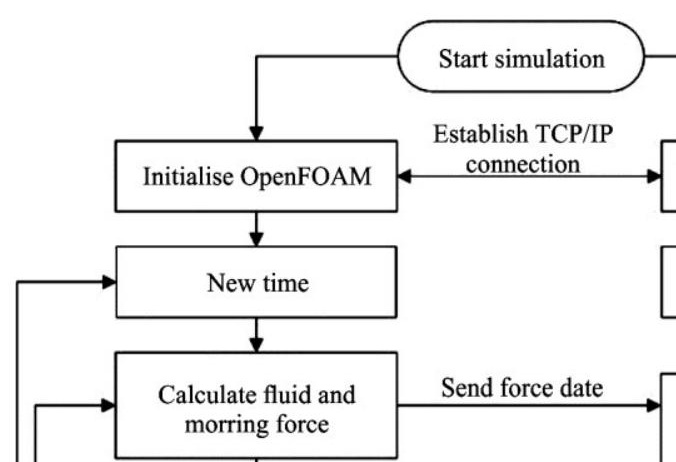


established to exchange force and motion data between the CFD and MBD models ${ }^{[24]}$.

As illustrated in Fig. 6, the surface grid of the structure in the CFD model is decomposed into several small patches, each of which corresponds to a beam node in the MBD model. A centre is defined for every patch in the CFD grid with the same kinematics as its associated beam node in the MBD model via motion data exchange. On the other hand, external fluid force and moment are firstly integrated over every patch of the CFD surface grid with respect to its patch centre and then transferred to MBDyn through force data exchange.

In order to maintain smooth transition between patches in the CFD model, a linear interpolation scheme ${ }^{[24]}$ is implemented to calculate position of surface grid points using kinematics from surrounding patch centres in the following way:

$$
\boldsymbol{X}=\xi\left(\boldsymbol{X}_{i}+\boldsymbol{R}_{i} \boldsymbol{d}_{i}\right)+(1-\xi)\left(\boldsymbol{X}_{i+1}+\boldsymbol{R}_{i+1} \boldsymbol{d}_{i+1}\right)
$$

where $\boldsymbol{X}$ represents position of point or patch centre; $\boldsymbol{R}$ denotes transformation matrix of patch centre due to rotation; $\boldsymbol{d}$ is distance vector pointing from patch centre to point; $\xi \in[0,1]$ stands for normalised point location between surrounding patch centres.
Figure 7 shows the coupling procedure adopted in the present FSI analysis tool. When a fully coupled simulation is performed, both OpenFOAM and MBDyn run simultaneously as individual computer processes. Exchange of motion and force data between the two codes is achieved by adopting a TCP/IP communication protocol and implementing an interface library in OpenFOAM which serves as a bridge connecting the flow and structural solvers.

\subsection{Validation studies}

The fully coupled analysis tool developed in the present work consists of various modules and features essential for FOWT simulations, ranging from aerodynamics, hydrodynamics, mooring dynamics to FSI. A series of test cases has been simulated and analysed in previous studies to validate these components individually. Specifically, numerical simulations of a NREL Phase VI wind turbine under various wind conditions were performed and results were compared with experimental data available to validate the capability of the developed tool in modelling wind turbine aerodynamics $^{[17]}$. A DeepCwind semi-submersible platform designed for FOWT applications was also investigated numerically and the hydrodynamic modelling feature of the present tool was validated against wave tank tests ${ }^{[2]}$. Additionally, the coupling procedure between OpenFOAM and MBDyn was validated by studying flow induced oscillations of a flexible cantilever beam in the wake of a fixed square $^{[23]}$.

In order to validate the dynamic mooring analysis module recently developed in the present tool, a 
flexible hanging line is studied with its principal properties listed in Table 1. The line is $170 \mathrm{~m}$ long when there is no tension force applied. The top end of the line is $5 \mathrm{~m}$ below water surface and it is thus completely submerged in water with infinite depth to exclude effects of free surface and seabed. The tangential component is set to 0 for both drag and added mass coefficients for simplicity.

Table 1 Properties of a flexible hanging line

\begin{tabular}{cc}
\hline Properties & Value \\
\hline Total unstretched length $L / \mathrm{m}$ & 170 \\
Horizontal distance between two ends $L_{H}$ & 100 \\
Vertical distance between two ends $L_{V} / \mathrm{m}$ & 50 \\
Top end below water surface $D_{T} / \mathrm{m}$ & 5 \\
Diameter $d / \mathrm{m}$ & 0.396 \\
Mass per unit length $m / \mathrm{kg} \cdot \mathrm{m}^{-1}$ & 165 \\
Weight per unit length in water $($ buoyancy & 410 \\
excluded) $w / \mathrm{N} \cdot \mathrm{m}^{-1}$ & 500 \\
Extensional stiffness $E A / \mathrm{MN}$ & 0 \\
Tangential drag coefficient $C_{D T}$ & 1 \\
Normal drag coefficient $C_{D N}$ & 0 \\
Tangential added mass coefficient $C_{A T}$ & 1 \\
Normal added mass coefficient $C_{A N}$ & 68 \\
Number of segments $n$ &
\end{tabular}

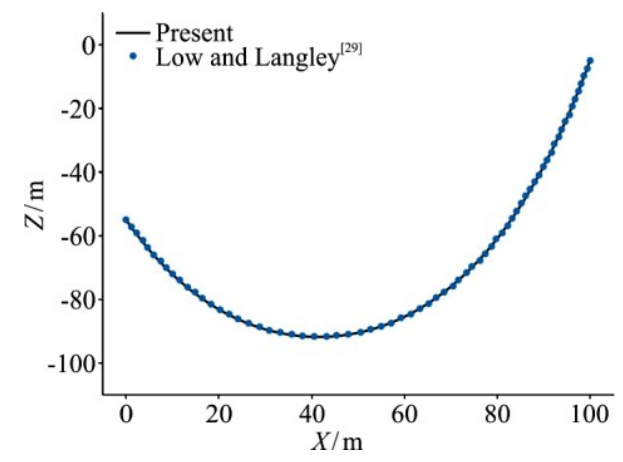

Fig. 8 (Color online) Static shape of flexible hanging line

Figure 8 plots the shape of the line predicted by the present tool while it is static in still water without considering hydrodynamic loadings. Due to the gravitational force, the line hangs between its two ends. Results from another lumped-mass-based approach by Low and Langley ${ }^{[30]}$ are also displayed in Fig. 8 for comparison. Good agreement between the two sets of data is achieved, demonstrating the good accuracy of the quasi-static solver developed in the mooring system analysis module. The static results of shape and line tension are later used by the dynamic code as initial conditions.

Subsequently, a prescribed sinusoidal motion with an amplitude of $10 \mathrm{~m}$ and a motion period of $27 \mathrm{~s}$ is imposed to the top end of the line to study its dynamic characteristics while the bottom end is fixed. Figure 9 shows the time history plots of the tension force measured at the top end of the line. Strong nonlinearity can be observed from the results, where secondary peaks are clearly shown in addition to the primary peaks. This can be explained by the slack or hanging configuration of the line. Figure 9 also demonstrates that the results obtained by Low and Langley ${ }^{[30]}$ agree well with those predicted using the present dynamic analysis code. As a result, the mooring system analysis module developed in the FSI tool is successfully validated.

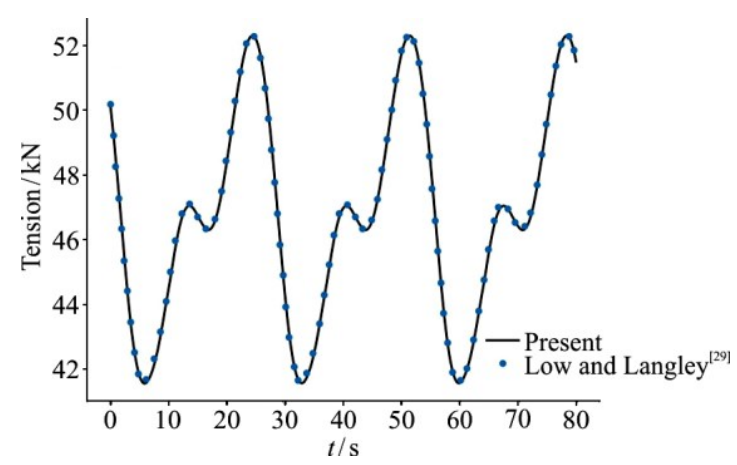

Fig. 9 (Color online) Time history of tension force at top end of flexible hanging line

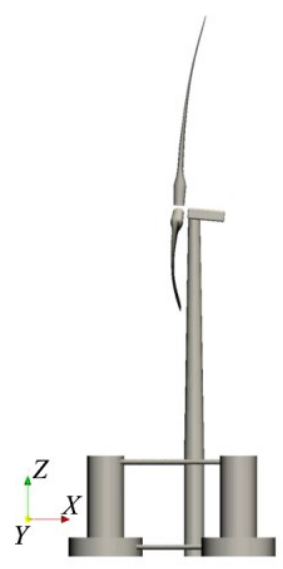

(a)

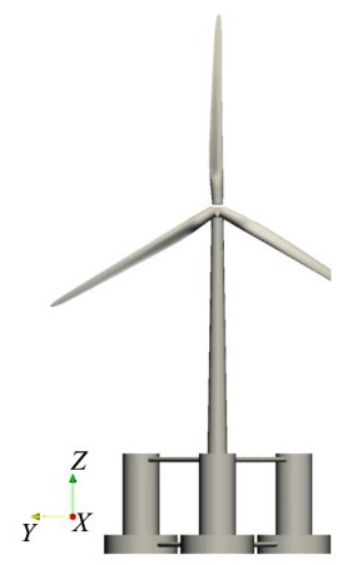

(b)
Fig. 10 (Color online) Geometry of an OC4 semi-submersible FOWT with flexible blades

\section{Model description}

In the present work, a fully coupled analysis is carried out for an OC4 FOWT consisting of a NREL$5 \mathrm{MW}$ wind turbine with three flexible blades and a three-column semi-submersible platform, as illustrated in Fig. 10. The principal properties of the model are mostly the same as those described in a previous work regarding the same OC4 FOWT with rigid blades ${ }^{[22]}$. However, due to the blade deflections induced by wind, 
a shaft tilt angle of $5^{\circ}$ is applied to the turbine along with a blade pre-cone angle of $2.5^{\circ}$ to maintain the clearance between the turbine blades and the tower. Additionally, the overhang of the turbine is set to $5 \mathrm{~m}$ and a nacelle is added to the top of the tower.

The computational mesh generated for the CFD simulation is shown in Fig. 11. Mesh refinement is applied near the free surface to simulate wave propagation and wave-structure interactions. Cells are also clustered in the vicinity of turbine blade tip and root regions as well as in the wake to capture complex vortex evolution and turbine-wake interactions. It is worth pointing out that a fully coupled FOWT simulation with flexible turbine blades is computationally expensive. Due to limited computational resource available, the grid density in the present study is adjusted to achieve a cell count of $3 \times 10^{6}$. As a result, the primary objectives of this study are to demonstrate the capabilities of the numerical tool developed for this project and to analyse the interaction between different components of the floating system in a qualitative manner.

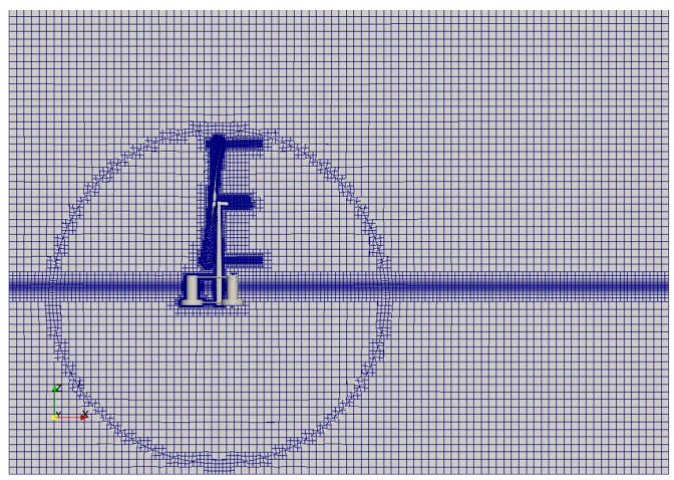

Fig. 11 (Color online) Computational grid for OC4 FOWT

In order to establish the structural model in MBDyn, 49 geometrical nodes (24 three-node beam elements) are adopted for each of the three blades, as exemplified in Fig. 12. A hub node is defined for the turbine hub, and the nodes at blade root are clamped to it. A platform node is also introduced to represent the floating platform in the fully couple case. In addition, a separate static or ground node is used as a reference node for other nodes, resulting in a total number of 150 nodes. The hub node is forced to rotate relative to the platform node along its rotation axis at a specified turbine rotation speed using an axial rotation joint while the platform node is allowed to move with respect to the static ground node. As a result, the wind turbine moves with the floating platform when it responds to the excitation forces from environmental waves and wind.

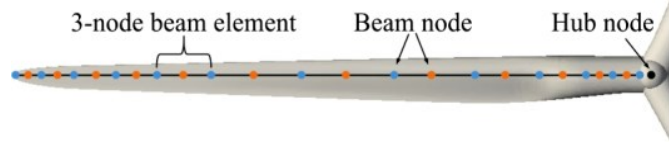

Fig. 12 (Color online) Illustration of structural model for a turbine blade ${ }^{[24]}$

The floating structure is equipped with a mooring system comprising three catenary lines. The properties of the mooring system previously listed in Liu et al. ${ }^{[22]}$ are adopted for the present study. However, as dynamic mooring line analysis is carried out in the simulation, additional information such as the inertia and drag coefficients of the mooring lines in tange- ntial and normal directions is required. The data estimated by Hall and Goupe ${ }^{[31]}$ to validate their developed lumpedmass mooring line model against MARIN's model tests ${ }^{[3]}$ is utilised in this study and summarised in Table 2 .

Table 2 Inertia and drag coefficients for dynamic mooring line analysis

\begin{tabular}{cc}
\hline Properties & Value \\
\hline Tangential drag coefficient $C_{D T}$ & 0.213 \\
Normal drag coefficient $C_{D N}$ & 1.08 \\
Tangential added mass coefficient $C_{A T}$ & 0.269 \\
Normal added mass coefficient $C_{A N}$ & 0.865 \\
\hline
\end{tabular}

In this study, the OC4 semi-submersible FOWT is subject to regular waves and uniform wind. Table 3 lists the environmental conditions adopted for the present simulation. The Stokes 2nd order wave theory is employed with an amplitude of $3.79 \mathrm{~m}$ and a period of $12.1 \mathrm{~s}$. The uniform wind speed is $11.4 \mathrm{~m} / \mathrm{s}$, and the turbine rotates with a constant rotor speed of 12.1 RPM and zero blade pitch angle. Wind and wave are aligned in the positive $X$ direction defined in Fig. 10.

Table 3 Environmental conditions for OC4 FOWT

\begin{tabular}{cc}
\hline Properties & Value \\
\hline Wave amplitude/m & 3.79 \\
Wave period $/ \mathrm{s}$ & 12.1 \\
Wind speed $/ \mathrm{m} \cdot \mathrm{s}^{-1}$ & 11.4 \\
Rotor speed $/ \mathrm{RPM}$ & 12.1 \\
Rotor rotation period $/ \mathrm{s}$ & 4.96 \\
Blade pitch angle $/{ }^{\circ}$ & 0 \\
\hline
\end{tabular}

\section{Results and discussion}

Numerical results obtained from the fully coupled aero-hydro-mooring-elastic simulation of the OC4 semi-submersible FOWT are presented and analysed from the following aspects: platform hydro- dynamics, mooring dynamics, wind turbine aerody- namics and blade elasticity. The popular engineering tool FAST v8 is also adopted for comparison with the present tool. 
The control system used in FAST v8 to regulate wind turbine operation is disabled in the present study.

\subsection{Platform hydrodynamics}

Figure 13 shows time history curves of surge and pitch responses under the combined wind/wave condition predicted by the present fully coupled CFDMBD tool and the engineering tool FAST v8. Periodic results for one complete wave period are depicted and extracted for further analysis. It is easily seen that the wind turbine significantly impacts the motion responses of the platform. Specifically, the surge motion obtained using the present tool has a maximum value of $10.56 \mathrm{~m}$ with a mean position of $8.2 \mathrm{~m}$, and the maximum angle for pitch motion reaches $4.35^{\circ}$ with an equilibrium value of $3.47^{\circ}$. Results from FAST v8 also show similar trends for surge (max: $10.51 \mathrm{~m}$, mean: $8.28 \mathrm{~m}$ ) and pitch $\left(\max : 4.18^{\circ}\right.$, mean: $3.24^{\circ}$ ). This can be associated with the significant aerodynamic thrust generated by the wind turbine under the present environmental condition.

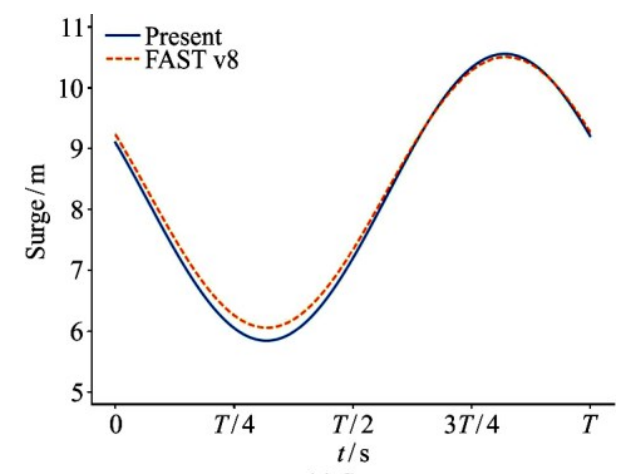

(a) Surge

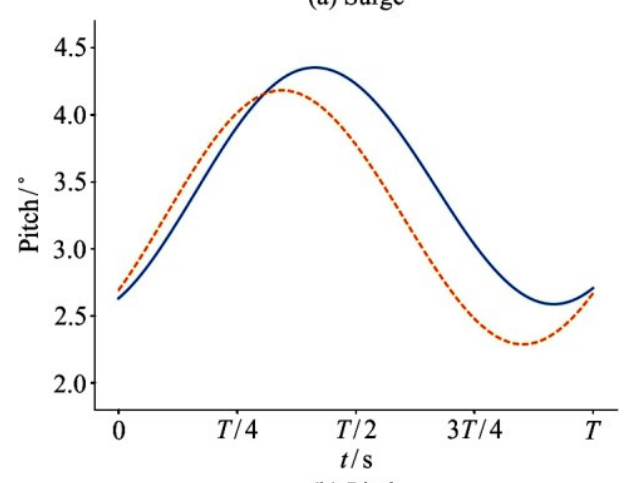

(b) Pitch

Fig. 13 (Color online) Time history of platform motion responses

Table 4 compares RAO results of the two platform motion responses predicted by the two tools. In order to further analyse the effects of wind turbine aerodynamic loadings on the platform, data from a previously studied case ${ }^{[22]}$ (referred to as "wave only") is also listed, where the wind turbine is not modelled. Compared to the wave only condition, variations are present for results from the present tool under the combined wind/wave condition. For example, surge RAO increases from 0.5965 for the wave only condition by $4.27 \%$ to 0.622 for the combined wind/wave condition. Nevertheless, considering that the computational mesh for the present study is relatively coarse as mentioned in Section 2, difference between results under the two conditions is deemed small, which indicates that the impacts of the wind turbine with flexible blades on platform motion RAO are negligible for the investigated case.

Table 4 Motion RAO comparison between present tool and FAST v8

\begin{tabular}{cccc}
\hline RAO & Wave only & Present & FAST v8 \\
\hline Surge & 0.5965 & $0.6220(+4.27 \%)$ & $0.5879(-1.44 \%)$ \\
Pitch/ & 0.2470 & $0.2329(-5.71 \%)$ & $0.2499(+1.17 \%)$ \\
${ }^{\circ} \cdot \mathrm{m}^{-1}$ & & & \\
\hline
\end{tabular}

Meanwhile, similar results from FAST v8 also suggest that the calibrated FAST model for the OC4 semi-submersible FOWT is able to accurately predict its hydrodynamic responses under the current operating condition of combined regular waves and uniform wind However, it should be noted that the potential flow theory adopted by engineering tools like FAST v8 to deal with platform hydrodynamics is based on linear assumptions. Furthermore, drag coe- fficients required to take into account viscous damping have to be extracted from experimental tests and are assumed to be constant for all flow conditions. It is thus expected that these engineering tools might be inadequate for highly nonlinear problems, such as extreme wave conditions and large motion responses, which the present CFD-based tool is capable of.

\subsection{Mooring dynamics}

Time history data of the tension force measured at the fairlead of the mooring line in head wave direction over one wave cycle is plotted in Fig. 14 for the two tools. Dynamic analysis is carried out for the mooring system in both simulations (labelled with dynamic). The dynamic mooring line analysis module MoorDyn incorporated in FAST v8 adopts the same lumped mass model as the present tool. The two curves are very similar in terms of trend and magni- tude. Particularly, the temporary plateau between $T / 4, T / 2$, possibly due to the inclusion of non- linear force terms in the dynamic model, is predicted in both simulations. Quantitative results listed in

Table 5 Comparison for mooring line tension with various methods

\begin{tabular}{cccc}
\hline & Wave only static & Present static & FAST v8 \\
\hline $\mathrm{RAO} / \mathrm{kN} \cdot \mathrm{m}^{-1}$ & 27.546 & 51.586 & $48.680(-)$ \\
Mean $/ \mathrm{MN}$ & 1.146 & 1.634 & $1.622(-0$ \\
Maximum $/ \mathrm{MN}$ & 1.251 & 1.830 & $1.806(-1$ \\
\hline
\end{tabular}


Table 5 also reveal that the difference between RAO predicted by the two tools is $4.6 \%$ and that discrepancies in mean and maximum tension are $-2.97 \%$, $-1.66 \%$, respectively, which further validates the dynamic mooring line modelling feature implemented in the present tool. It is also noted that the fairlead tension force is largely out of phase with the platform surge motion, which can be attributed to the additional dynamic DOFs, i.e., acceleration and velocity, introduced in the lumped mass model.

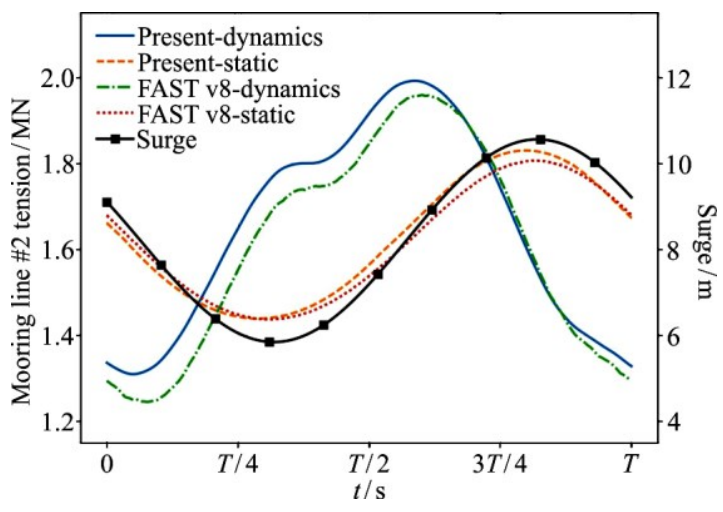

Fig. 14 (Color online) Time history of mooring line tension

In order to assess the difference between the two different approaches in modelling mooring lines, i.e. quasi-static and dynamic methods, tension force results from simulations with quasi-static mooring line analysis (labelled with static) are also included in Fig. 14 and Table 5. Quasi-static results from the two tools agree reasonably well with a maximum difference of $-5.63 \%$ in tension RAO. Comparison between data obtained with quasi-static and dynamic approaches in present simulations shows that tension RAO significantly increases from $51.586 \mathrm{kN} / \mathrm{m}$ by about $75 \%$ to $90.036 \mathrm{kN} / \mathrm{m}$, which demonstrates the importance of a dynamic model in predicting mooring line tension and structural strength design. Meanwhile, the difference between mean tension predictions is small, i.e., 1.04\%. However, Fig. 14 shows that unlike the dynamic model, the tension force obtained with the quasi-static model is mostly in phase with the platform surge motion, which is likely due to lack of dynamic effects.

Results from the previously studied wave only case $^{[22]}$, where the quasi-static method was adopted, are also listed in Table 5. By comparing the present case with quasi-static mooring line analysis to the wave only case, it is found that mean tension increases from 1.146 $\mathrm{MN}$ to $1.634 \mathrm{MN}$ as a result of the wind turbine thrust force pushing the platform away from its equilibrium position. Besides, tension RAO from the present quasi-static study is $87.27 \%$ larger than that in the wave only case, which can be explained by the nonlinear relationship between the mooring restoring force and the large surge motion response.

\subsection{Wind turbine aerodynamics}

Figure 15 shows time history curves of aerodynamic power of the wind turbine within one wave cycle from the present tool and FAST v8. A series of sudden drops can be clearly observed in the power curves, which is caused by the presence of the turbine tower when turbine blades pass in front of it, also known as tower interference or tower shadow effects. Both tools predict similar magnitude for these sudden decreases, i.e., approximately $300 \mathrm{~kW}$ for power.

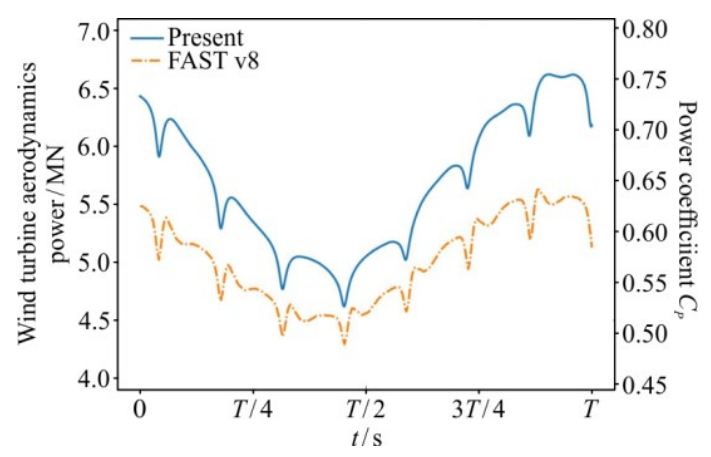

Fig. 15 (Color online) Time history of wind turbine aerodynamic performance

Due to the platform motion responses, particularly surge and pitch, it can be found that wind turbine aerodynamic power oscillates over the time range of one wave cycle. Although oscillation is present in both curves, the amplitude of power oscillation predicted by the present tool is different from that obtained with FAST v8. The current high-fidelity tool predicts a variation of about $2 \mathrm{MW}$ measured from maximum to minimum while results from FAST v8 show an oscillation of about $1.3 \mathrm{MW}$, which is $50 \%$ smaller. Similar findings about smaller oscillation amplitudes from engineering tools like FAST v8 compared to CFD-based solvers were also pointed out by Quallen et al. ${ }^{[32]}$, Tran and $\mathrm{Kim}^{[20]}$ in their respective FOWT simulations. This is likely due to the many empirical models adopted in these engineering tools, such as the dynamic wake model and skewed wake model. These models were originally formulated for fixed-bottom wind turbines and might not be suitable for complex conditions experienced by wind turbines installed on floating platforms. Specifically, in addition to the rotor tilt and blade precone angles, a positive equilibrium 
angle is present for the pitch motion, which forces the wind turbine to operate in skewed wake under constantly changing flow condi- tions due to periodic platform surge and pitch motion responses.

Figure 16 illustrates four snapshots for fluid flow over one incident wave cycle. The vortical structures generated at blade tip and root areas are clearly visible, which are represented by the contour of second invariant of the rate of strain tensor $Q(Q=0.05)$ and coloured by the axial component of fluid flow velocity $U_{x}$. The platform surge and pitch motions induced in this study lead to clear interactions between the wind turbine and its wake, which can be demonstrated by the variation in the distance between two adjacent vortex tubes in the lower part of the skewed wake (highlighted in black rectangles). It is very difficult for the empirical wake models adopted by FAST v8 to accurately take these interactions into account, which partly explains the difference between wind turbine aerodynamic performance from the two tools. Sec- tional view of fluid flow at the mid-plane of the computational domain is also exhibited in Fig. 16 to analyse the spatial and temporal variation of velocity field influenced by platform motions. The black circles annotated in Fig. 16 clearly show impacts of platform motion on incoming wind. Furthermore, air flow is also affected by wave propagation as indicated by decrease of wind speed above wave crests, which emphasises the coupling between wind and wave in FOWT simulations. Application of the present CFD method enables detailed investigation into the com- plex fluid flow around the FOWT via visualisation, which cannot be achieved by engineering tools like FAST v8.

\subsection{Blade elasticity}

Figure 17 compares time history data of blade tip deflection in the flapwise direction within one wave cycle predicted by the present tool and FAST v8. The BeamDyn module based on a nonlinear beam theory is implemented in FAST v8 to deal with deformation of flexible structures. Unlike results presented in the previous work $^{[24]}$ for the case with prescribed platform surge motion, both curves show rapid changes in blade deformation due to the influence of additional platform pitch motion induced by waves and non-zero tilt angle applied. Tower interference effects are also clearly visible in blade deflection results, indicated by the two sudden drops in the curves as annotated in Fig. 17. When the blade passes in front of the turbine tower, the aerodynamic force exerted upon the blade decreases rapidly, leading to reduced blade defor- mation. Results obtained with FAST v8 are generally smaller than predictions from the present CFD-MBD tool, which is in accordance with the turbine aerodynamic loadings in Fig. 15.

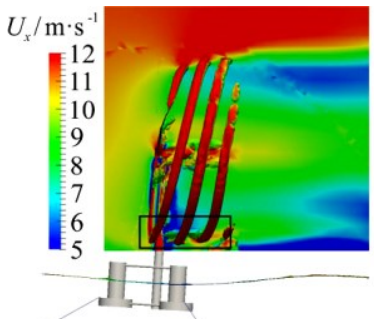

(a) $t=T / 8$

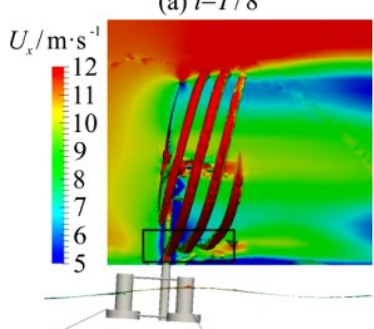

(c) $t=5 T / 8$

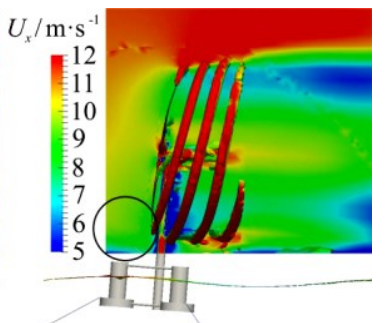

(b) $t=3 T / 8$

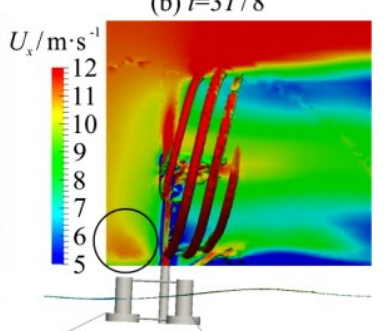

(b) $t=7 T / 8$
Fig. 16 (Color online) Vortex contour and flow field at midplane coloured by axial velocity $U_{x}$

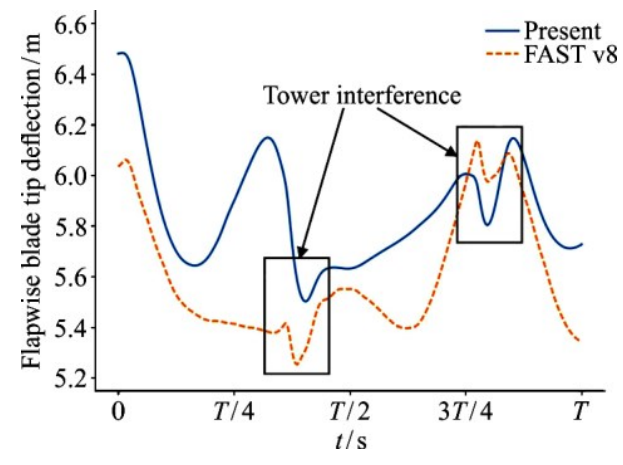

Fig. 17 (Color online) Time history of flapwise blade tip deflection

Figure 18 demonstrates time history curves of the bending moment at blade root in the flapwise direction within one wave cycle. The variation of blade root bending moment closely resembles that of blade tip deflection shown in Fig. 17, including the sudden drops due to tower interference. It should be noted that in addition to frequency associated with wind turbine rotation and tower interference, the platform motion responses experienced by the FOWT also introduce a frequency related to the incident waves in the temporal change of blade root bending moment. For the current case with moderate wave height, a considerable amount of variation is present in blade root bending moment. It is thus reasonable to expect even more significant changes in structural loading when the FOWT operates under conditions with higher wave height than the present study, which may lead to severe structural problems and therefore stresses the importance of taking into account plat- form motion responses in blade structural design for FOWTs. 


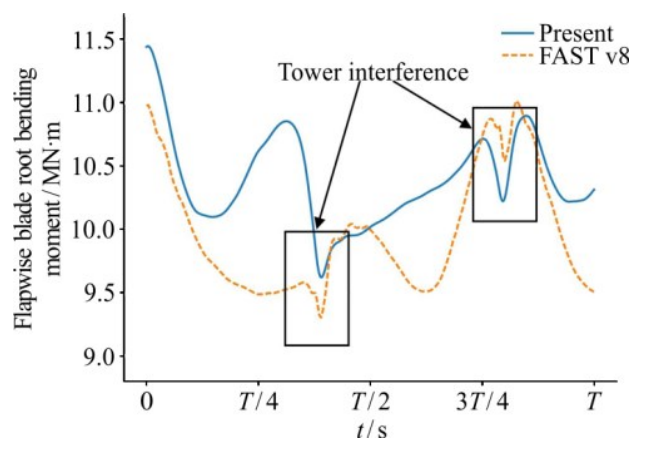

Fig. 18 (Color online) Time history of flapwise blade root bending moment

\section{Conclusions}

In this paper, a fully coupled high-fidelity tool based on a CFD-MBD approach is developed for aerohydro-mooring-elastic analysis of FOWTs. The open source CFD toolbox OpenFOAM is utilised to model fluid flow, including turbulence modelling and free surface capturing via the VOF method. A wave modelling module is incorporated for wave generation and damping in a numerical wave tank. The open source MBD code MBDyn is employed for calculating the structural response of a multibody system comprising rigid and flexible components. A mesh motion solver is developed in OpenFOAM to handle complex mesh movement in FOWT simulations due to global rigid body motions, such as platform 6DOF motion and wind turbine rotation, and local flexible body deformation, i.e. turbine blade deflection. A mooring system analysis module is also implemented in OpenFOAM with both quasi-static and dynamic methods. The two separate codes OpenFOAM and MBDyn are coupled via an interface library implemented to exchange loading and response data.

With the fully coupled CFD-MBD tool implemented in this work, high-fidelity aero-hydromooring-elastic analysis is carried out for an OC4 semi-submersible FOWT with flexible blades under a combined wind/wave condition. Results from the present study are analysed and compared to data obtained with the engineering too FAST v8 from various perspectives, including platform hydrodynamic responses, mooring system dynamics, wind turbine aerodynamic performance and blade structural dynamics. Interactions among different components of the floating system are also investigated.

Impacts of wind turbine aerodynamics with elastic blades on platform surge and pitch responses in regular waves are limited to their equilibrium posi- tions while motion RAOs are generally unaffected. Mooring line tension RAO predicted via a dynamic approach is $75 \%$ larger than data obtained with a quasi-static method, underlying the importance of model fidelity in FOWT mooring system design. Comparing turbine aerodynamic power from the two tools reveals that the engineering tool under-predicts the oscillation amplitude by $50 \%$ due to the many empirical models adopted in FAST v8, which cannot consider complex FSI caused by platform motions. Visualisation of the flow field obtained via the CFD approach clearly shows the interactions between the wind turbine and its wake, the influence of platform motion on the wind field as well as the impacts of wave propagation on air flow. Tower interference and platform motions lead to rapid oscillations in blade tip deflection and root bending moment, which should be taken into account during the structural design of FOWT blades.

The high-fidelity CFD-MBD tool developed in this work presents a way to help understand the complex interactions between fluid flow and an FOWT as well as the impacts of different parts of the system on each other under various conditions. Although the present study focuses on uniform wind and regular wave, it can be extended in future research to more complex environmental conditions, such as irregular and extreme waves, nonuniform wind with shear velocity profiles and initial explicit turbulence, as well as current, in which the simplified models employed by engineering tools like FAST v8 are insufficient for accurate predictions.

\section{Acknowledgements}

The first author would like to acknowledge $\mathrm{Mr}$ Christophe Peyrard from Électricité de France (EDF) for generously providing insightful suggestions and comments to this work and for kindly offering access to the Athos HPC facility in EDF. This work was supported by the National Natural Science Foundation of China (Grant No. U1806229).

\section{References}

[1] WindEurope. The European offshore wind industry: Key trends and statistics 2016 [R]. Brussels, Belgium: WindEurope, 2017.

[2] Roddier D., Cermelli C., Aubault A. et al. WindFloat: A floating foundation for offshore wind turbines [J]. Journal of Renewable and Sustainable Energy, 2010, 2(3): 033104.

[3] Coulling A. J., Goupee A. J., Robertson A. N. et al. Validation of a FAST semi-submersible floating wind turbine numerical model with DeepCwind test data [J]. Journal of Renewable and Sustainable Energy, 2013, 5(2): 023116.

[4] Skaare B., Nielsen F. G., Hanson T. D. et al. Analysis of measurements and simulations from the Hywind Demo floating wind turbine [J]. Wind Energy, 2015, 18(6): 11051122.

[5] EWEA. Deep water: The next step for offshore wind energy [R]. Brussels, Belgium: European Wind Energy 
Association, 2013.

[6] Bachynski E. E., Kvittem M. I., Luan C. et al. Wind-wave misalignment effects on floating wind turbines: Motions and tower load effects [J]. Journal of Offshore Mechanics and Arctic Engineering, 2014, 136(4): 041902.

[7] Karimirad M., Michailides C. Dynamic analysis of a braceless semisubmersible offshore wind turbine in operational conditions [J]. Energy Procedia, 2015, 80: 2129.

[8] Oguz E., Clelland D., Day A. H. et al. Experimental and numerical analysis of a TLP floating offshore wind turbine [J]. Ocean Engineering, 2018, 147: 591-605.

[9] Li L., Liu Y., Yuan Z. et al. Wind field effect on the power generation and aerodynamic performance of off- shore floating wind turbines [J]. Energy, 2018, 157: 379-390.

[10] Sebastian T., Lackner M. A. Development of a free vortex wake method code for offshore floating wind turbines [J]. Renewable Energy, 2012, 46: 269-275.

[11] Liu F., Chen J., Qin H. Frequency response estimation of floating structures by representation of retardation functions with complex exponentials [J]. Marine Structures, 2017, 54: 144-166.

[12] Nematbakhsh A., Olinger D. J., Tryggvason G. A nonlinear computational model of floating wind turbines [J]. Journal of Fluids Engineering, 2013, 135(12): 121103.

[13] Tran T. T., Kim D. H. The coupled dynamic response computation for a semi-submersible platform of floating offshore wind turbine [J]. Journal of Wind Engineering and Industrial Aerodynamics, 2015, 147: 104-119.

[14] Nematbakhsh A., Bachynski E. E., Gao Z. et al. Comparison of wave load effects on a TLP wind turbine by using computational fluid dynamics and potential flow theory approaches [J]. Applied Ocean Research, 2015, 53: 142154.

[15] Subbulakshmi A., Sundaravadivelu R. Heave damping of spar platform for offshore wind turbine with heave plate [J]. Ocean Engineering, 2016, 121: 24-36.

[16] Tran T. T., Kim D. H. A CFD study into the influence of unsteady aerodynamic interference on wind turbine surge motion [J]. Renewable Energy, 2016, 90: 204-228.

[17] Liu Y., Xiao Q., Incecik A. et al. Investigation of the effects of platform motion on the aerodynamics of a floating offshore wind turbine [J]. Journal of Hydrody-namics, 2016, 28(1): 95-101.

[18] Wu C. H. K., Nguyen V. T. Aerodynamic simulations of offshore floating wind turbine in platform-induced pitching motion [J]. Wind Energy, 2017, 20(5): 835-858.

[19] Quallen S., Xing T. CFD simulation of a floating offshore wind turbine system using a variable-speed generatortorque controller [J]. Renewable Energy, 2016, 97: 230-242.

[20] Tran T. T., Kim D. H. Fully coupled aero-hydrodynamic analysis of a semi-submersible FOWT using a dynamic fluid body interaction approach [J]. Renewable Energy, 2016, 92: 244-261.

[21] Leble V., Barakos G. Demonstration of a coupled floating offshore wind turbine analysis with high-fidelity methods [J]. Journal of Fluids and Structures, 2016, 62: 272-293.

[22] Liu Y., Xiao Q., Incecik A. et al. Establishing a fully coupled CFD analysis tool for floating offshore wind turbines [J]. Renewable Energy, 2017, 112: 280-301.

[23] Liu Y., Xiao Q., Incecik A. A coupled CFD/multibody dynamics analysis tool for offshore wind turbines with aeroelastic blades [C]. 36th International Conference on Ocean, Offshore and Arctic Engineering, Trondheim, Norway, 2017.

[24] Liu Y., Xiao Q., Incecik A. et al. Aeroelastic analysis of a floating offshore wind turbine in platform-induced surge motion using a fully coupled CFD-MBD method [J]. Wind Energy, 2019, 22(1): 1-20.

[25] Cao H., Wan D. Development of multidirectional nonlinear numerical wave tank by naoe-FOAM-SJTU solver [J]. International Journal of Ocean System Engineering, 2014, 4(1): 52-59.

[26] Shen Z. R., Wan D. C. An irregular wave generating approach based on naoe-FOAM-SJTU solver [J]. China Ocean Engineering, 2016, 30(2): 177-192.

[27] Menter F. R. Review of the shear-stress transport turbulence model experience from an industrial perspective [J]. International Journal of Computational Fluid Dynamics, 2009, 23(4): 305-316.

[28] Hirt C. W., Nichols B. D. Volume of fluid (VOF) method for the dynamics of free boundaries [J]. Journal of Computational Physics, 1981, 39(1): 201-225.

[29] Ghiringhelli G. L., Masarati P., Mantegazza P. Multibody implementation of finite volume $\mathrm{C}(0)$ beams [J]. AIAA Journal, 2000, 38(1): 131-138.

[30] Low Y. M., Langley R. S. Dynamic analysis of a flexible hanging riser in the time and frequency domain [C]. 25th International Conference on Offshore Mechanics and Arctic Engineering, Hamburg, Germany, 2006, 161-170.

[31] Hall M., Goupee A. Validation of a lumped-mass mooring line model with DeepCwind semisubmersible model test data [J]. Ocean Engineering, 2015, 104: 590-603.

[32] Quallen S., Xing T., Carrica P. et al. CFD Simulation of a floating offshore wind turbine system using a quasi-static crowfoot mooring-line model [J]. Journal of Ocean and Wind Energy, 2014, 1(3): 143-152. 